\title{
TOTALLY ACCRETIVE OPERATORS
}

\author{
RALPH DELAUBENFELS
}

(Communicated by Paul S. Muhly)

\begin{abstract}
Let $A$ be a (possibly unbounded) linear operator on a Banach space. We show that, when $A$ generates a uniformly bounded strongly continuous semigroup $\left\{e^{-t A}\right\}_{t \geq 0}$, then $A^{2}$ generates a bounded holomorphic semigroup (BHS) of angle $\theta$ if and only if $A$ generates a BHS of angle $\theta / 2+\pi / 4$. We show that each power of $A$ generates a uniformly bounded strongly continuous semigroup if and only if $A$ generates a BHS of angle $\pi / 2$ if and only if each power of $A$ generates a BHS of angle $\pi / 2$. If $A$ is a linear operator on a Hilbert space, then each power of $A$ generates a strongly continuous contraction semigroup if and only if $A$ is positive selfadjoint.
\end{abstract}

I. Introduction. When the (possibly unbounded) linear operator, $A$, on a Banach space, generates a bounded, strongly continuous semigroup, $\left\{e^{-t A}\right\}_{t>0}$, it is natural to ask when powers of $A$ might also generate such semigroups. In this paper, we discover that quite a bit may be determined about $A$, when these semigroups exist. Even if we merely require that both $A$ and $A^{2}$ generate bounded strongly continuous (not necessarily holomorphic) semigroups, it is necessary that $A$ generate a BHS (bounded holomorphic semigroup-see Definition 5) of angle $\pi / 4$ (Theorem 8 ). If we also ask that $A^{2}$ generate a BHS, then our necessary conditions also become sufficient (see Theorem 8 ).

We show that, in order that each power of $A$ generates a bounded strongly continuous (not necessarily holomorphic) semigroup, it is necessary and sufficient that $A$ generate a BHS of angle $\pi / 2$. This answers an open question in [4]. This also implies the following surprising equivalence. Each power of $A$ generates a bounded strongly continuous semigroup if and only if each power of $A$ generates a BHS of angle $\pi / 2$ (Theorem 11). This identifies the class of operators that generate a BHS of angle $\pi / 2$ as being of some special interest.

When $A$ acts on a Hilbert space, and we make the stronger requirement that each power of $A$ generates a strongly continuous contraction semigroup, then we find that $A$ must be a selfadjoint operator, with nonnegative spectrum (Theorem 13). This first appeared in [3]. The terminology "totally $m$-accretive", meaning that each power generates a strongly continuous contraction semigroup, is also from [3].

A natural generalization of Theorem 13 would be that a totally $m$-accretive operator is a scalar-type spectral operator. Example 14 gives a counterexample to this. The operator is $d^{2} / d x^{2}$, with maximal domain, on $L^{p}[-\pi, \pi]$, where $1<$ $p<\infty, p \neq 2$. A generalization of scalar-type spectral operators that has received much attention recently is well-bounded operators (see [5]). In Open Question 15,

Received by the editors February 27, 1987.

1980 Mathematics Subject Classification (1985 Revision). Primary 47B44. 
we consider whether an operator with nonnegative spectrum is well-bounded if and only if it is totally $m$-accretive, with respect to an equivalent norm.

In [4], we showed that, when $A$ generates a BHS of sufficiently large angle, then some powers of $A$ also generate bounded holomorphic semigroups. Hence, a part of this paper is the converse of results in [4].

A result analogous to Theorem 11 appears in [2], where it is shown that, if $B$ is a bounded operator such that, for all $n, B^{n}$ is Hermitian $\left( \pm i B^{n}\right.$ is accretive), then $B$ is a spectral operator of scalar type (compare with Example 14, and Open Question 15).

We begin with a lemma about square roots of operators (Lemma 7-see Definition 6). The results stated in the Abstract are in Theorems 8, 11, and 13. We conclude with some open questions and some comments about "natural" generalizations that are not true.

Finally, we would like to make a comment about terminology. Whether one deals with accretive or dissipative ( $A$ is dissipative if $(-A)$ is accretive) operators depends on whether one wishes to write a semigroup as $e^{t B}$ (generated by the dissipative operator $B$ ) or $e^{-t A}$ (generated by the accretive operator $A$ as in [8]). We chose to say "accretive" because our main results (Theorems 11 and 13) are more easily stated.

All operators are linear, on a Banach space, usually called " $X$ ". We will write " $D(C)$ " for the domain of the operator $C$.

We first need some preliminary definitions.

DEFINITION 1. We say that an operator, $A$, is accretive if its numerical range $\equiv\{\varphi(A x) \mid x$ is in the domain of $A, \varphi(x)=\|x\|,\|\varphi\|=1\}$ is contained in the right half-plane $\equiv\{$ complex $z \mid \operatorname{Re}(z) \geq 0\}$.

DEFINITION 2 . We say that $A$ is $m$-accretive if it generates a strongly continuous contraction semigroup $\left\{e^{-t A}\right\}_{t \geq 0}$ (see [8]). This is equivalent to the spectrum and numerical range of $A$ both being contained in the right half-plane.

DEFINITION 3. We say that $A$ is accretive-equivalent if there exists an equivalent norm with respect to which $A$ is accretive.

DEFINITION 4. We say that $A$ is m.e.-accretive if it generates a bounded strongly continuous semigroup $\left\{e^{-t A}\right\}_{t \geq 0}$. This is equivalent to saying that there exists an equivalent norm with respect to which $A$ is $m$-accretive.

DEFINITION 5. We say that $A$ generates a bounded holomorphic semigroup (BHS) of angle $\theta(0<\theta \leq \pi / 2)$ if $A$ is m.e.-accretive, and the map $t \rightarrow e^{-t A}$ extends to a function holomorphic on

$$
S_{\theta} \equiv\{\text { complex } z|| \arg (z) \mid<\theta\},
$$

and bounded and strongly continuous on $\bar{S}_{\psi}$, whenever $\psi<\theta$.

To state our results on square roots in their most natural generality, we would like to make the following extension of Definitions 1-5.

Definition 6. If $\theta<\pi$, we say that the operator $A$ is of type $\theta$ if $A$ is densely defined and the spectrum of $A$ is contained in $\bar{S}_{\theta}$, and for all $\psi>\theta$, there exists finite $M_{\psi}$ such that, for all $z$ outside $S_{\psi}$,

$$
\left\|(z-A)^{-1}\right\| \leq M_{\psi} /|z| .
$$


An operator generates a BHS of angle $\theta$ if and only if it is of type $(\pi / 2-\theta)$. An m.e.-accretive operator is of type $\pi / 2$.

In [1], and in other places where fractional powers of closed operators are constructed, the class of operators of type less than $\pi$ is considered.

LEMMA 7. Suppose $A$ is of type $\theta<\pi$. Then there exists unique m.e.-accretive $B$ such that $B^{2}=A$. $B$ is of type $\theta / 2$.

PROOF. A simple consequence of [1, Theorem 6.1] is that any operator of type $<\pi$ has a unique m.e.-accretive square root. In the proof, it is shown that this square root generates a BHS.

Let $B$ be this m.e.-accretive square root of $A$. We wish to show that $B$ generates a BHS of angle $(\pi / 2-\theta / 2)$ (see Definitions 6 and 5 ).

Let $F \equiv\left\{\right.$ real $\varphi \mid e^{i \varphi} B$ generates a BHS $\}$. It is clear that $F$ is open. The following argument shows that $G \equiv(-(\pi / 2-\theta / 2),(\pi / 2-\theta / 2))-F$ is also open.

Suppose $\psi$ is in $G$. Since $e^{2 \psi i} A$ is of type $\theta+2|\psi|<\pi$, it has a unique m.e.accretive square root, $C$, which generates a BHS. There exists $\varepsilon>0$ such that $e^{ \pm i \varphi} C$ generates a BHS whenever $0<\varphi<\varepsilon$, and $(\psi-\varepsilon, \psi+\varepsilon)$ is contained in the union of $G$ with $F$. We claim that $(\psi-\varepsilon, \psi+\varepsilon)$ is disjoint from $F$. If not, then there exists $\varphi$ such that $|\varphi|<\varepsilon, e^{i(\varphi+\psi)} B$ generates a BHS. Since $\left(e^{i(\varphi+\psi)} B\right)^{2}=$ $\left(e^{i \varphi} C\right)^{2}=e^{2(\psi+\varphi) i} A$, uniqueness of square roots says that $e^{i(\varphi+\psi)} B=e^{i \varphi} C$, so that $e^{i \psi} B=C$. This says that $\psi \in F$, which is a contradiction. Thus $(\psi-\varepsilon, \psi+\varepsilon)$ is disjoint from $F$, so that $G$ is open.

Since both $F$ and $(-(\pi / 2-\theta / 2),(\pi / 2-\theta / 2))-F$ are open, $(-(\pi / 2-\theta / 2),(\pi / 2-$ $\theta / 2)$ ) is contained in $F$.

Thus, whenever $|\psi|<\pi / 2-\theta / 2, e^{ \pm i \psi} B$ generates a BHS. It remains to show that $\left\{\left\|e^{-z B}\right\| \mid z \in S_{\psi}\right\}$ is bounded. This follows by a simple compactness argument, since, for all $\varphi \in[-\psi, \psi]$, because $e^{i \varphi} B$ generates a BHS, there exists $\varepsilon>0$ such that $\left\{\left\|e^{-z B}\right\||| \arg (z)-\varphi \mid<\varepsilon\right\}$ is bounded.

THEOREM 8. Suppose $A$ and $A^{2}$ are m.e.-accretive. Then $A$ generates a BHS of angle $\pi / 4$. $A^{2}$ generates a BHS of angle $\theta$ if and only if $A$ generates a BHS of angle $\pi / 4+\theta / 2(0<\theta \leq \pi / 2)$.

PROOF. Lemma 7 says that $A$ is of type $\pi / 4$, and that, if $A^{2}$ is of type $\pi / 2-\theta$, then $A$ is of type $\pi / 4-\theta / 2$ (see Definition 6 ). The converse of the second assertion is in [4], and in the proof of Proposition 2, in [6].

COROllaRY 9. Suppose $A^{2^{k}}$ is m.e.-accretive, for $k=0,1,2, \ldots, n$. Then $A$ generates a $B H S$ of angle $\pi\left(1-\left(\frac{1}{2}\right)^{n}\right) / 2$.

ProOF. By induction and Theorem $8, A^{2^{(n-i)}}$ generates a BHS of angle $\pi\left(1-\left(\frac{1}{2}\right)^{i}\right) / 2$, for $i=0,1,2, \ldots, n$.

DEFINITION 10. We say that $A$ is totally accretive (from [3]), if, for all natural numbers $n, A^{n}$ is accretive (see Definition 1). Totally $m$-accretive, totally accretiveequivalent, and totally m.e.-accretive are defined similarly.

Note that being totally m.e.-accretive is not the same as being totally $m$-accretive in an equivalent norm; the latter property is equivalent to $\sup \left\{\left\|e^{-t p(A)}\right\| \mid p\right.$ is a 
polynomial with positive coefficients $\}$ being finite. Theorems 11 and 13 imply that, at least on a Hilbert space, being totally $m$-accretive is fundamentally different than being totally m.e.-accretive, since the class of generators of bounded holomorphic semigroups of angle $\pi / 2$ is larger than the class of positive selfadjoint operators. (See also Example 14 and Open Question 15.)

THEOREM 11. The following are equivalent.

(a) $A$ is totally m.e.-accretive.

(b) A generates a $B H S$ of angle $\pi / 2$.

(c) For all $n, A^{n}$ generates a $B H S$ of angle $\pi / 2$.

PrOOF. (a) implies (b) follows from Corollary 9. (b) implies (c) is in [4, Corollary 5]. (c) implies (a) is obvious.

The proof in [8, Theorem 3.35] gives the following (compare with Lemma 7).

LEMMA 12 (FROM [8]). If $A$ is m-accretive on a Hilbert space, then there exists unique m-accretive $B$ such that $B^{2}=A$. If $A$ has spectrum and numerical range contained in $S_{\theta}$, then $B$ has spectrum and numerical range contained in $S_{\theta / 2}$.

Lemma 12 is false in an arbitrary Banach space (see Example 14).

The following first appeared in [3].

THEOREM 13. Suppose $A$ is an operator on a Hilbert space. Then the following are equivalent.

(a) $A$ is $m$-accretive and totally accretive.

(b) $A$ is totally m-accretive.

(c) $A$ is positive selfadjoint.

ProOF. (a) implies (c): For any $n$, since $(1+A)$ is invertible, so is $(1+A)^{n}$. Since $(1+A)^{n}$ is also accretive, $(1+A)^{n}$ is $m$-accretive. Arguing as in Corollary 9 , using Theorem 12 instead of Theorem 7 , we deduce that the spectrum and numerical range of $(1+A)$ are contained in $[0, \infty)$. Since $A$ is accretive, the spectrum and numerical range of $A$ are also contained in $[0, \infty)$, that is, $A$ is positive selfadjoint. (c) implies (b) implies (a) is obvious.

EXAMPLE 14. An operator on a Hilbert space that is selfadjoint is a scalar-type spectral operator. We give here an example of a totally $m$-accretive operator that is not a scalar-type spectral operator. (Compare with Theorem 13.)

Let $X$ be $L^{p}[-\pi, \pi]$, where $1<p<\infty$, with the following equivalent norm. Let $h_{k}(t) \equiv e^{i k t}, \hat{f}(k) \equiv$ the $k$ th Fourier coefficient of $f$, and

$$
(F(N)) f=\sum_{k=-N}^{N} \hat{f}(k) h_{k} .
$$

Then our norm is

$$
\|f\| \equiv \sup _{N}\|(F(N)) f\|_{p}
$$

This is an equivalent norm, since the Fourier series of an $L^{p}$-function converges in $L^{p}$. Note that, with respect to this norm, $\|F(N)\|=1$, for any $N$.

Let $A$ be $\left(-d^{2} / d x^{2}\right)$, with maximal domain. The following calculation will show that $\left\|e^{-s A^{n}}\right\|$ equals 1 , for any $n, s$. 


$$
\begin{aligned}
\left\|e^{-s A^{n}} f\right\| & =\left\|\sum_{k=-\infty}^{\infty} e^{-s k^{2 n}} \hat{f}(k) h_{k}\right\| \\
& =\left\|\sum_{k=0}^{\infty}(F(k)-F(k-1)) f e^{-s k^{2 n}}\right\| \\
& =\left\|\sum_{k=0}^{\infty}(F(k) f)\left(e^{-s(k+1)^{2 n}}-e^{-s k^{2 n}}\right)\right\| \quad \text { (summation by parts) } \\
& \leq \sup _{N}\|F(N) f\| \sum_{k=0}^{\infty}\left(e^{-s k^{2 n}}-e^{-s(k+1)^{2 n}}\right) \\
& =\|f\| .
\end{aligned}
$$

Thus $A$ is totally $m$-accretive. But unless $p=2, A$ is not a scalar-type spectral operator.

This example also shows that Lemma 12 is false in an arbitrary Banach space, since otherwise the proof of Theorem 13 would show that, if $A$ is totally $m$-accretive, then all powers of $A$ have nonnegative spectrum and numerical range (since all powers are totally $m$-accretive). This, in turn, can be shown to imply that $A$ is a scalar-type spectral operator (see [2], for the bounded case).

OPEN QUESTION 15. An operator, $A$, on a reflexive Banach space, $X$, is well bounded on $[0, \infty)$ if there exists a bounded family of projection, $\{F(t)\}_{t \geq 0}$, on $X$, as in [5, Definition 16.8], such that

$$
A x=\lim _{N \rightarrow \infty}\left(N F(N) x-\int_{0}^{N} F(t) x d t\right),
$$

with the domain of $A$ equal to the set of $x$ for which that limit exists.

Note that integration by parts shows that a positive selfadjoint operator on a Hilbert space, or more generally, a positive scalar-type spectral operator on a Banach space, is well bounded on $[0, \infty)$.

Essentially the same argument as in Example 14 shows that, if $A$ is well bounded on $[0, \infty)$, then there exists an equivalent norm with respect to which $A$ is totally $m$-accretive.

Is the converse true, that is, is a totally $m$-accretive operator well bounded? (Compare with Theorems 13 and 11.)

OPEN QUESTION 16 (FROM [3]). Suppose $A$, an operator on a Hilbert space, is totally accretive, but not necessarily $m$-accretive, with $D\left(A^{n}\right)$ dense, for all $n$. Is $A$ positive symmetric?

\section{BIBLIOGRAPHY}

1. A. V. Balakrishnan, Fractional powers of closed operators and the semigroups generated by them, Pacific J. Math. 10 (1960), 419-437.

2. E. Berkson, A characterization of scalar type operators on reflexive Banach spaces, Pacific J. Math. 13 (1963), 365-373.

3. P. Chernoff, On totally accretive operators, unpublished note, 1978.

4. R. deLaubenfels, Powers of generators of holomorphic semigroups, Proc. Amer. Math. Soc. 99 (1987), 105-108.

5. H. R. Dowson, Spectral theory of linear operators, Academic Press, 1978. 
6. J. A. Goldstein, Some remarks on infinitesimal generators of analytic semigroups, Proc. Amer. Math. Soc. 22 (1969), 91-93.

7.

8. T. Kato, Perturbation theory for linear operators, Springer-Verlag, 1966.

9. A. Pazy, Semigroups of linear operators and applications to partial differential equations, SpringerVerlag, 1983.

10. M. Reed and B. Simon, Methods of modern mathematical physics, part II, Academic Press, 1975.

11. K. Yosida, Functional analysis (6th ed.), Springer-Verlag, 1980.

Department of Mathematics, Ohio University, Athens, Ohio 45701 